Article

\title{
Modeling and Simulation of a Wave Energy Converter INWAVE
}

\author{
Seung Kwan Song ${ }^{1}$, Yong Jun Sung ${ }^{2}$ and Jin Bae Park ${ }^{1, *}$ \\ 1 Department of Electrical and Electronic Engineering, Yonsei University, 50 Yonsei-ro, \\ Seoul 03722, Korea; nomadnosad@gmail.com \\ 2 INGINE Inc., Changdo Building, 395-2 Cheonho-daero, Seoul 02633, Korea; yjsung@ingine.co.kr \\ * Correspondence: jbpark@yonsei.ac.kr; Tel.: +82-2-2123-2773
}

Academic Editors: Yongheng Yang and Frede Blaabjerg

Received: 7 November 2016; Accepted: 16 January 2017; Published: 19 January 2017

\begin{abstract}
INGINE Inc. developed its own wave energy converter (WEC) named INWAVE and has currently installed three prototype modules in Jeju Island, Korea. This device is an on-shore-type WEC that consists of a buoy, pulleys fixed to the sea-floor and a power take off module (PTO). Three ropes are moored tightly on the bottom of the buoy and connected to the PTO via the pulleys, which are moving back and forth according to the motion of the buoy. Since the device can harness wave energy from all six degrees of movement of the buoy, it is possible to extract energy efficiently even under low energy density conditions provided in the coastal areas. In the PTO module, the ratchet gears convert the reciprocating movement of the rope drum into a uni-directional rotation and determine the transmission of power from the relation of the angular velocities between the rope drum and the generator. In this process, the discontinuity of the power transmission occurs and causes the modeling divergence. Therefore, we introduce the concept of the virtual torsion spring in order to prevent the impact error in the ratchet gear module, thereby completing the PTO modeling. In this paper, we deal with dynamic analysis in the time domain, based on Newtonian mechanics and linear wave theory. We derive the combined dynamics of the buoy and PTO modules via geometric relation between the buoy and mooring ropes, then suggest the ratchet gear mechanism with the virtual torsion spring element to reduce the dynamic errors during the phase transitions. Time domain simulation is carried out under irregular waves that reflect the actual wave states of the installation area, and we evaluate the theoretical performance using the capture width ratio.
\end{abstract}

Keywords: wave energy converter; point absorber; on-shore energy absorbing unit; capture width ratio

\section{Introduction}

Since industrialization, the use of fossil fuel energy has increased exponentially. Consequently, the awareness and concern for the entirety life on Earth is growing due to side effects, such as global warming. As a result, the importance of renewable energy is on the rise, and energy sources that use wind, sun and geothermal heat are at the commercialization stage [1].

Among the sources for renewable energy that await commercialization in the near future is wave energy [2]. Although the idea of wave energy has existed for a long time, the commercialization has been slower than other sources of renewable energy. Unlike sunlight or wind that have relatively uniform energy flow, wave energy has a reciprocating motion, high energy density with slow speed and seasonal variations in heights and periods [3]. As a result, wave energy converters (WECs) must have a complicated mechanism, solidity and flexibility corresponding to each attribute.

In addition, since waves generally have higher energy density at a deep water depth and the density becomes lesser as they get closer to the shore, it is appropriate to install the WECs in the distant sea, which has a deep water depth [4]. Hence, the accessibility is poor, and a long sub-sea transmission 
line is needed. As the accessibility is low, WECs should be more robust so that less maintenance is required. To reduce the proportion of the cable installation cost in the capital expenditure of the WEC, a large-capacity wave energy farm should be built. Consequently, it requires more complete initial verification than other renewable energy sources for commercialization [5].

One of the aforementioned disadvantages of wave energy is that the area of high wave energy density is far away from the on-grid. However, depending on the location, it can actually work as an advantage. One of these locations would be remote islands that are off-grid and therefore do not receive electricity from the mainland. Diesel generators are used for sourcing electricity in these islands. Since the power production unit price of a diesel generator is higher than that of the mainland, renewable energy sources in the islands are cost competitive. This has been represented in the example of Samsø Island in Denmark [6]. Wave energy can be seen as the most suitable option for creating an energy self-sufficient island.

In consideration of such a situation, the wave power company INGINE has created a wave power generator called INWAVE. Currently, there are three full-scale prototype devices installed in Jeju Island as a test run [7]. The company seeks to start commercial operations in the latter half of 2016. This device, a tight moored moving body-type WEC, shares similar concepts with Fred Olsen's Lifesaver WEC, in that the buoy has six degrees of freedom for movement and is connected to the sea-floor via stiff mooring ropes. While the Lifesaver [8] is installed at a medium water depth of about $50 \mathrm{~m}$, INWAVE is installed along the coast. The INWAVE device can follow the theoretical analysis of the Lifesaver since the basic concept is similar to this device. In the papers dealing with the Lifesaver, however, there is no modeling description for the multi-mooring and multi-degree motion of the WEC, but a point absorber modeling with heave motion is only described. In [9], meanwhile, a multi-point tight moored body-type WEC is presented. However, only the frequency domain analysis is applied to the optimal control theory. Hence, there are limits to using this modeling approach directly.

In order to realize the modeling of the INWAVE device, it is necessary to derive the kinematic relations of mooring ropes between the buoy and PTO (power take off) module and to build up the ratchet gear modeling in the PTO module. The ratchet gear is a mechanical element that converts the reciprocating motion of the mooring rope into a uni-directional rotation to the generator. This process is determined by the relationship between the angular velocities of the rope drum and the generator. As the ratchet gear connects or disconnects the rope drum with the generator, the dynamic modeling of the PTO module has discontinuity that causes the systemic errors and instability. To solve this problem, we introduce the PTO modeling, which does not cause discontinuous change by applying the virtual torsion spring element to the ratchet gear. We present the INWAVE modeling including this PTO module based on the Newtonian mechanics and linear wave theory (LWT) $[10,11]$. Finally, we carry out simulations under JONSWAPirregular waves in various wave periods, to demonstrate the characteristics of the device.

\section{Explanation of the Device}

As shown in Figure 1, INWAVE (INGINE Inc., Seoul, Korea) consists of the buoy and the power take off (PTO) module, which is located on land. The buoy is discus shaped and is connected to ropes by three points on the bottom, and this rope is connected to the PTO module located on land via a pulley fixed to the sea-floor. The PTO module consists of a counterweight, the gearbox module (which includes the ratchet gears) and the generator. Individual ropes that are connected to the buoy are connected to a counterweight. The counterweight provides tension on the rope consistently, and this tension gives the buoy dynamic stability. 


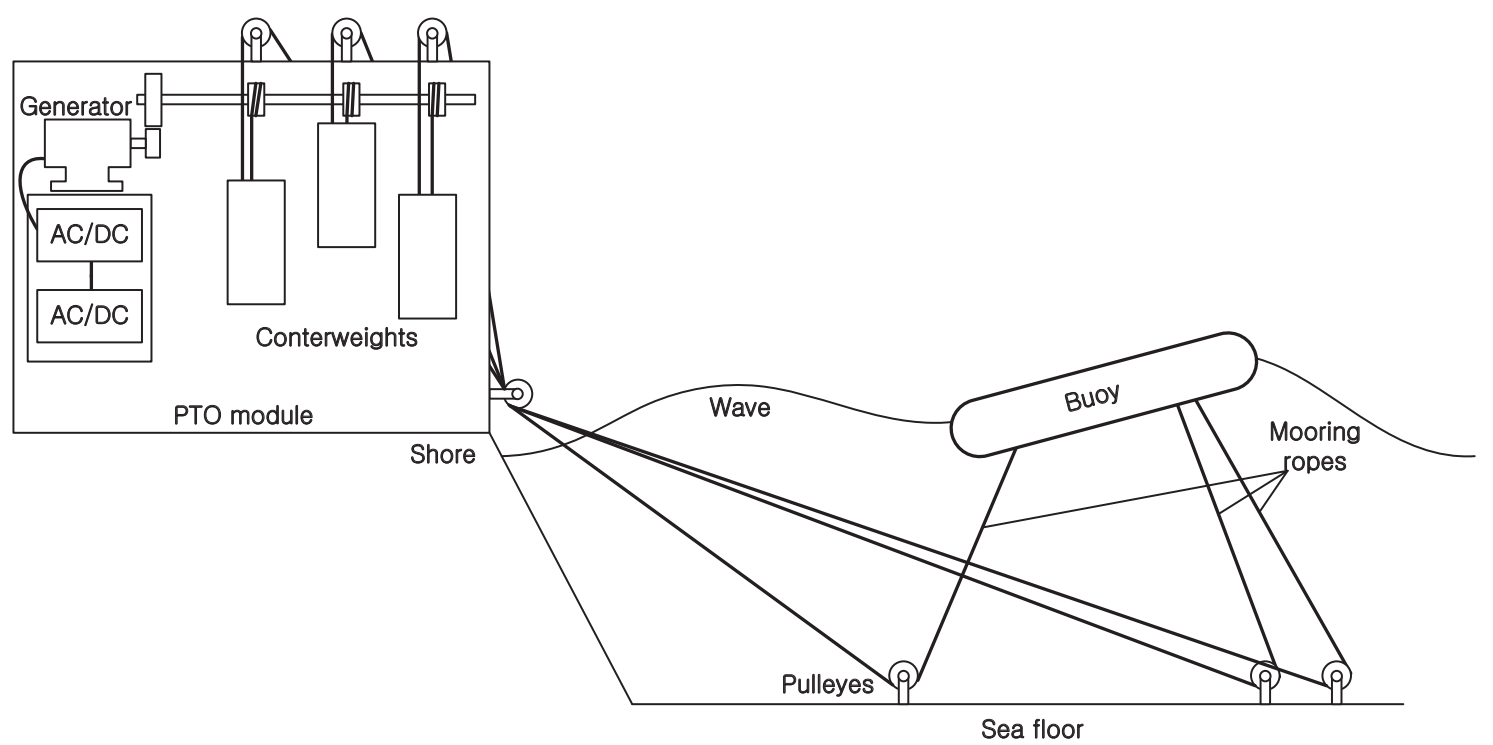

Figure 1. Schematics of the INWAVE device model configuration.

In calm water conditions, the buoy maintains equilibrium due to the weight, buoyancy and tension from the weights. The buoy goes through a complex movement that can be divided into heave, surge, sway, roll, pitch and yaw, as the wave passes over it. Depending on the movement of the buoy, ropes are pulled, and the counterweights either rise or lower. Each rope is wound on the rope drum, and the rope drum is connected to the generator. The ratchet gear, which is mounted on the rope drum, only sends the power to the generator when the buoy pulls the ropes, and as such, the counterweight is rising. Since the motion of the three ropes is not in the same phase, the time point for the sending of the power by the individual rope drum is different, which results in a mechanical smoothing effect. The generator uses a $20-\mathrm{kW}$ permanent magnet synchronous generator (Yaskawa, Tokyo, Japan) and is linked to the grid through an AC/DC converter (LSIS, Gyeonggi-do, Korea) and a DC / AC inverter (LSIS, Gyeonggi-do, Korea). As shown in Figure 2, currently, three modules are installed on Jeju Island's northern coastal region for a test run. It was installed in September of 2015 and seeks to start operating commercially in the latter half of 2016.

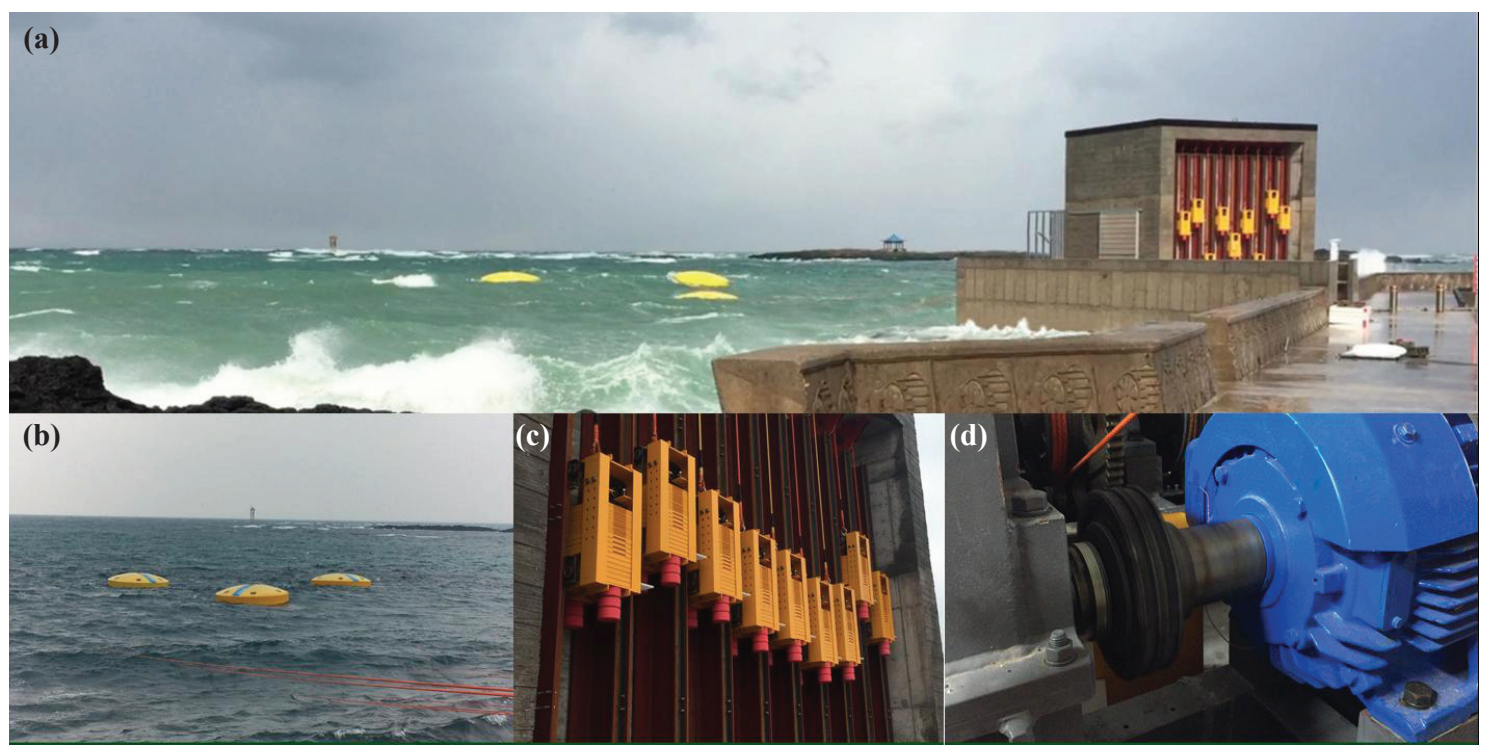

Figure 2. INWAVE prototype installed in the northern port of Jeju Island: (a) panorama of the device; (b) buoys; (c) counterweights; and (d) electric generator [7]. 


\section{Modeling of the Device's Dynamics}

\subsection{Buoy-Counterweight Modeling}

A buoy and PTO module are mechanically connected by the rope tensions that are applied by vector decomposition in each moving direction from the relationship between the buoy's posture and pulleys on the sea-bed. In this chapter, to derive this relationship easily, we assume a two-dimensional analysis of the device with two-mooring points. This analysis has the advantage that intuitive consideration can be made by matching the dimension of the traveling direction of the waves and the movement dimension of the mooring ropes. The two-dimensional analysis will be a comparative case that can prove the validity of the complex three-dimensional analysis results. For this reason, we assume a discus-shaped model with mooring points connected by two points that are away from the center of the buoy by $r$; as seen in Figure 3, even the actual device is a discus-shaped buoy with the mooring point connected by three different points. The buoy has a radius $R$ and is submerged a distance of $d$ since it is connected by the counterweight. For the sake of convenience, let us name the left and right mooring ropes as 1, 2, respectively, and the related variables will follow suit.

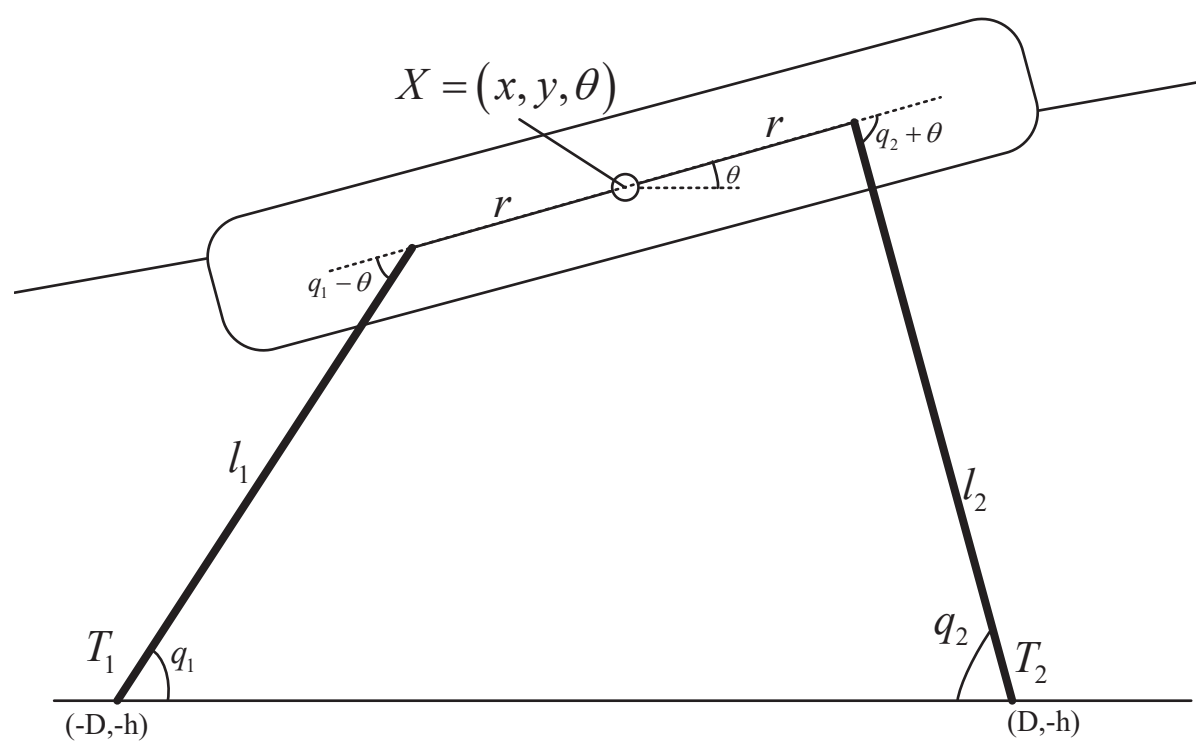

Figure 3. Geometries of the buoy and ropes.

The origin of the coordinate is on the water surface; the water depth is $h$; and fixed pulleys are away from the origin by $D$. In two dimensions, the buoy only moves in the directions of surge, heave and pitch, corresponding to the movements in the directions of $x, y$ and $\theta$, respectively. The buoy's posture is $X=[x, y, \theta]^{T}$; the lengths of the mooring ropes are $l_{1}$ and $l_{2}$, and the angles of them are $q_{1}$ and $q_{2}$, respectively. Then, the angles and lengths of the mooring ropes with the posture of the buoy have a geometric relation as follows:

$$
\begin{aligned}
& (-D,-h)+\left(l_{1} \cos q_{1}, l_{1} \sin q_{1}\right)+(r \cos \theta, r \sin \theta)=(x, y), \\
& (D,-h)+\left(-l_{2} \cos q_{2}, l_{2} \sin q_{2}\right)+(-r \cos \theta,-r \sin \theta)=(x, y) .
\end{aligned}
$$

The mooring ropes supply the buoy with tension $T_{1}$ and $T_{2}$ that are expressed as follows:

$$
\begin{aligned}
& T_{1}=m_{1} \ddot{l}_{1}+m_{1} g+f_{1}, \\
& T_{2}=m_{2} \ddot{l}_{2}+m_{2} g+f_{2} .
\end{aligned}
$$


$m_{1}$ and $m_{2}$ are the mass of the counterweights connected to Ropes 1 and 2, respectively, since this counterweight only moves vertically (acceleration of gravity $g$ ). $f_{1}$ and $f_{2}$ are the load forces related to the generator.

Then, the dynamic equation of the buoy is expressed as follows [10]:

$$
M_{b} \ddot{X}(t)+F_{r}(t)+K_{h} X(t)=F_{e}(t)+H\left[\begin{array}{ll}
T_{1} & T_{2}
\end{array}\right]^{T} .
$$

Here, $M_{b}$ is the mass and inertia matrix of buoy and the added mass effect, and $H$ is the geometric matrix related to how the rope's tensions $T_{1}$ and $T_{2}$ that affect the movement of the buoy. These are expressed as follows:

$$
\begin{gathered}
M_{b}=\operatorname{diag}\left[m_{b}+\mu_{x}(\infty), m_{b}+\mu_{y}(\infty), J_{b}+\mu_{\theta}(\infty)\right], \\
H=\left[\begin{array}{cc}
-\cos q_{1} & \cos q_{2} \\
-\sin q_{1} & -\sin q_{2} \\
r \sin \left(q_{1}-\theta\right) & -r \sin \left(q_{2}+\theta\right)
\end{array}\right] .
\end{gathered}
$$

$m_{b}$ and $J_{b}$ are the buoy's mass and the buoy's inertia in the direction of pitch movement, respectively. $\mu_{x}, \mu_{y}$ and $\mu_{\theta}$ are the added masses for the buoy's surge, heave and pitch motion, respectively. Diag[] is the diagonal matrix.

$F_{e}(t)$ is the excitation force vector as follows:

$$
F_{e}=\int_{-\infty}^{\infty}\left[\begin{array}{lll}
-f_{e x}(t-\tau)^{T} & f_{e y}(t-\tau)^{T} & f_{e \theta}(t-\tau)^{T}
\end{array}\right]^{T} \psi(\tau) d \tau,
$$

where:

$$
\begin{aligned}
& f_{e x}(t)=\frac{1}{2 \pi} \int_{-\infty}^{\infty} \hat{f}_{e x}(\omega) e^{j \omega t} d \omega, \\
& f_{e y}(t)=\frac{1}{2 \pi} \int_{-\infty}^{\infty} \hat{f}_{e y}(\omega) e^{j \omega t} d \omega, \\
& f_{e \theta}(t)=\frac{1}{2 \pi} \int_{-\infty}^{\infty} \hat{f}_{e \theta}(\omega) e^{j \omega t} d \omega
\end{aligned}
$$

$\psi$ is the wave elevation and $\hat{f}_{e}=\left[\hat{f}_{e x}, \hat{f}_{e y}, \hat{f}_{e \theta}\right]$ and $f_{e}=\left[f_{e x}, f_{e y}, f_{e \theta}\right]$ are the summation of the Froude-Krylov force and diffraction force vector (or named the excitation force) in the frequency domain and its kernel function in the time domain, respectively. We set the negative sign in front of $f_{e x}$ in (6) because the wave progresses to the left, as seen in Figure 1.

$F_{r}$ is the radiation force vector and is expressed as follows:

$$
F_{r}=\int_{-\infty}^{t}\left[f_{r x}(t-\tau)^{T} f_{r y}(t-\tau)^{T} f_{r \theta}(t-\tau)^{T}\right]^{T} \dot{X}(\tau) d \tau,
$$

where:

$$
\begin{aligned}
& f_{r x}(t)=\frac{2}{\pi} \int_{0}^{\infty}\left\{\hat{R}_{x}(\omega)+i \omega\left(\mu_{x}(\omega)-\mu_{x}(\infty)\right)\right\} \cos (\omega t) d \omega \\
& f_{r y}(t)=\frac{2}{\pi} \int_{0}^{\infty}\left\{\hat{R}_{y}(\omega)+i \omega\left(\mu_{y}(\omega)-\mu_{y}(\infty)\right)\right\} \cos (\omega t) d \omega \\
& f_{r \theta}(t)=\frac{2}{\pi} \int_{0}^{\infty}\left\{\hat{R}_{\theta}(\omega)+i \omega\left(\mu_{\theta}(\omega)-\mu_{\theta}(\infty)\right)\right\} \cos (\omega t) d \omega
\end{aligned}
$$

$f_{r}=\left[f_{r x}, f_{r y}, f_{r \theta}\right]$ is the radiation damping kernel function in the time domain. $\hat{R}=\left[\hat{R}_{x}, \hat{R}_{y}, \hat{R}_{\theta}\right]$ is the radiation damping in the frequency domain.

$K_{h}$ is the spring coefficient dependent on buoyancy. Since there is no buoyancy in the surge direction, it is zero. $k_{y}$ and $k_{\theta}$ are buoyancy in the $y$ and $\theta$ direction, respectively, and are defined as follows:

$$
K_{h}=\operatorname{diag}\left[0, k_{y}, k_{\theta}\right]
$$


where:

$$
\begin{aligned}
k_{y} & =\pi \rho_{w} g R^{2}, \\
k_{\theta} & =\frac{\pi}{4} \rho_{w} g R^{4} .
\end{aligned}
$$

Here, $\rho_{w}$ is the density of the seawater. $k_{\theta}$ is the spring constant when the buoy rotates by $\theta$ of the small displacement in the pitching direction and is obtained as follows:

$$
k_{\theta}=2 \rho_{w} g \int_{0}^{R} 2 r^{2} \sqrt{R^{2}-r^{2}} d r=\frac{\pi}{4} \rho_{w} g R^{4} .
$$

When substituting (1) and (2) into (3), we can obtain the combined dynamics of counterweights and the buoy, expressed as follows:

$$
\left\{M_{b}+H E_{1}\right\} \ddot{X}(t)+F_{r}(t)+K_{h} X(t)=F_{e x}(t)+H\left(E_{2}+E_{3}\right),
$$

where:

$$
\begin{array}{r}
E_{1}=\left[\begin{array}{ccc}
-m_{1} \cos q_{1} & -m_{1} \sin q_{1} & m_{1} r \sin \left(q_{1}-\theta\right) \\
m_{2} \cos q_{2} & -m_{2} \sin q_{2} & -m_{2} r \sin \left(q_{2}+\theta\right)
\end{array}\right], \\
E_{2}=\left[\begin{array}{c}
m_{1} g+f_{1} \\
m_{2} g+f_{2}
\end{array}\right], \\
E_{3}=\left[\begin{array}{l}
m_{1}\left\{r \dot{\theta}^{2} \cos \left(q_{1}-\theta\right)+l_{1} \dot{q}_{1}^{2}\right\} \\
m_{2}\left\{r \dot{\theta}^{2} \cos \left(q_{2}+\theta\right)+l_{2} \dot{q}_{2}^{2}\right\}
\end{array}\right] .
\end{array}
$$

\subsection{Power Take Off Module Modeling}

A buoy undergoes complex motions in the heave, surge and pitch directions in the two dimensions. While heave and pitch receive restoring force due to buoyancy, the restoring force in the surge direction is solely dependent on the tension of the ropes. The further away it gets from the origin and in the surge $(x)$ direction, the difference between angles $q_{1}$ and $q_{2}$ grows, leading it to return back to the origin.

Rope drums (or rope pulleys) connected to the ropes repeat the winding and unwinding with the rope's back and forth movements. In order to deliver the torques from pulleys to the one-way electrical generator, bi-directional winding motions should be converted into a uni-directional rotation using a ratchet gear as seen in Figure 4. The use of the ratchet mechanism divides the power transmission processes as follows: when the buoy pulls on the rope, the rope pulls on the counterweight, making it rise. Simultaneously, it provides the driving torque to the generator. Conversely, when the counterweight falls, the driving torque is not transmitted to the generator, and it only provides the restoring force to the buoy through the rope tension.

The reverse setting that driving torque is connected when the counterweight is falling is also possible. However, this mechanism is risky because unless the counterweight is sufficient, the load of the generator will result in the rope losing tension. Then, the rope can deviate from the pulley or drum, which can result in breaking of the rope. For this reason, the reverse setting requires careful consideration of the relationship between the generator load and the mass of the counterweight. In this paper, we deal only with the former ratchet mechanism, in which the driving torque is transmitted when the counterweight rises. 


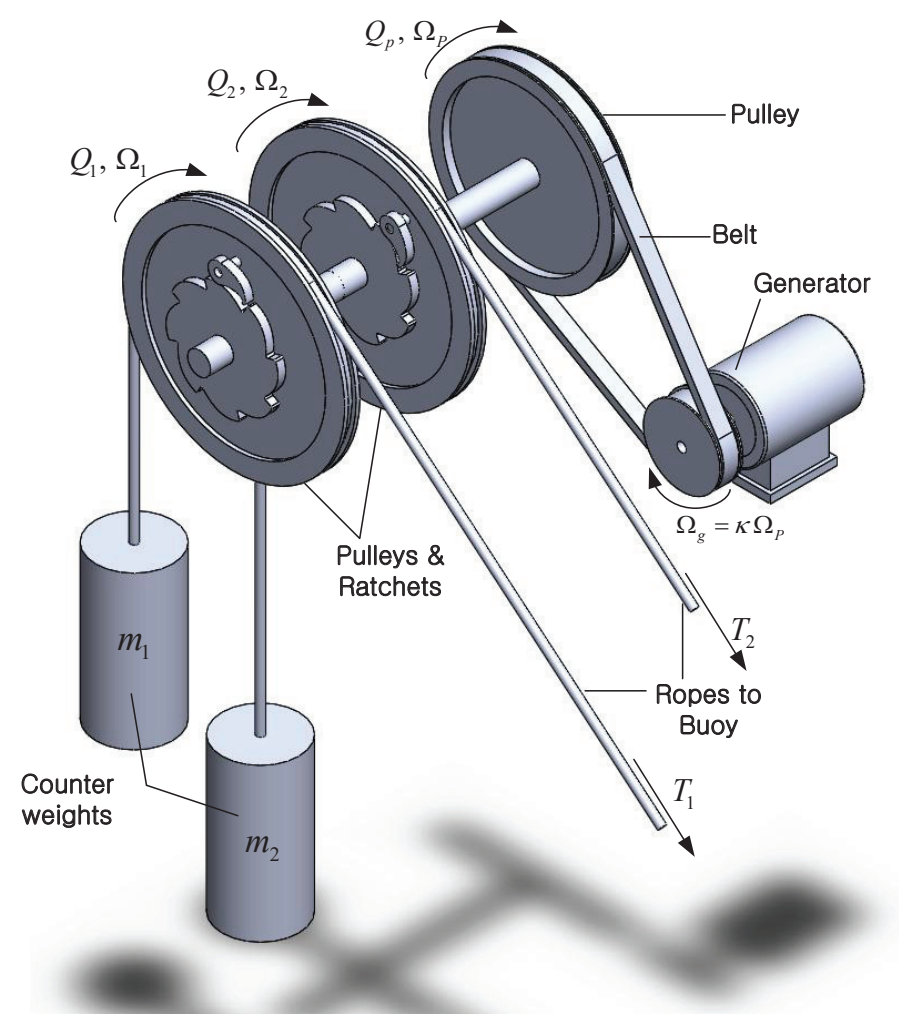

Figure 4. Power take off (PTO) module consisting of counterweights, pulleys, including ratchet gears, and the generator.

Figure 4 shows the overall configuration of the PTO module. The angular velocities of the pulleys that Ropes 1 and 2 are pulled over are $\omega_{1}\left(=\dot{l}_{1} / r_{1}\right)$ and $\omega_{2}\left(=\dot{l}_{2} / r_{2}\right)$, respectively, where $r_{1}$ and $r_{2}$ are the radii of the pulleys. The angular velocities of the generator and the pulley connected to it are $\omega_{g}$ and $\omega_{p}$, respectively, which have the relation of $\omega_{g}=\kappa \omega_{p}$ where $\kappa$ is the radius ratio between the pulley and generator. For numerical simplicity, it is assumed that the inertia of the pulleys and shafts can be ignored. Then, the dynamics of the generator can be expressed as follows:

$$
\kappa^{2} J_{g} \dot{\omega}_{p}=\tau_{1}+\tau_{2}-\kappa \tau_{g},
$$

where $J_{g}$ is the inertia of the generator rotor. $\tau_{1}, \tau_{2}$ and $\tau_{g}$ are the induced torques from Pulleys 1,2 and the generator, respectively. $\tau_{1}$ and $\tau_{2}$ have the following relation with $f_{1}$ and $f_{2}$ in (2) expressed as:

$$
\tau_{i}=r_{i} f_{i}, \quad \text { for } i=1,2
$$

We assume that induced torque from the generator $\tau_{g}$ is proportional to the angular velocity $\omega_{g}$ and is given as follows:

$$
\tau_{g}=b_{g} \omega_{g},
$$

where $b_{g}$ is the generator damping coefficient. Then, the instantaneous power output from the generator is:

$$
P_{g}=\tau_{g} \omega_{g}=b_{g} \omega_{g}^{2}=\kappa^{2} b_{g} \omega_{p}^{2} .
$$

Furthermore, the time-averaged output power is denoted by $\bar{P}\left(=\int P d t\right)$. Since the LWT is premised [11], the output power is proportional to the square of the wave amplitude. Hence, 
the output power per unit amplitude can be defined as $P^{*}=P /(\text { wave amplitude })^{2}$, and accordingly, time averaged power per unit amplitude is denoted as $\bar{P}_{g}^{*}$ [12].

The instantaneous power that the rope delivers to the pulley is:

$$
P_{i}=f_{i} \dot{l}_{i}=\tau_{i} \omega_{i}, \quad \text { for } i=1,2 .
$$

Additionally, in order to define how efficiently the power is transmitted from pulleys to the generator, we denote the ratchet efficiency as follows:

$$
\eta_{r}=\bar{P}_{g} /\left(\bar{P}_{1}+\bar{P}_{2}\right) \text {. }
$$

\subsection{Schematization of Ratchet Gears}

A ratchet gear modeling that rope pulley only transmits the torque with forward rotation to the generator while repeating the forward and reverse rotations is required and can be simply analyzed as follows:

The moment $\omega_{i}$ (for $i=1,2$ ) is greater than $\omega_{p}$; both are equivalent, and the torque $\tau_{i}$ is transmitted from the rope pulley to the generator. Thereafter, at the moment that the transmitted power $P_{i}\left(=\tau_{i} \omega_{i}\right)$ becomes negative, $\omega_{i}$ and $\omega_{p}$ are separated, and the torque is not delivered anymore. With the definitions of Phases 0 and 1 denoting the states for whether torque is transmitted or not, the ideal ratchet modeling can be schematized as shown in Figure 5.

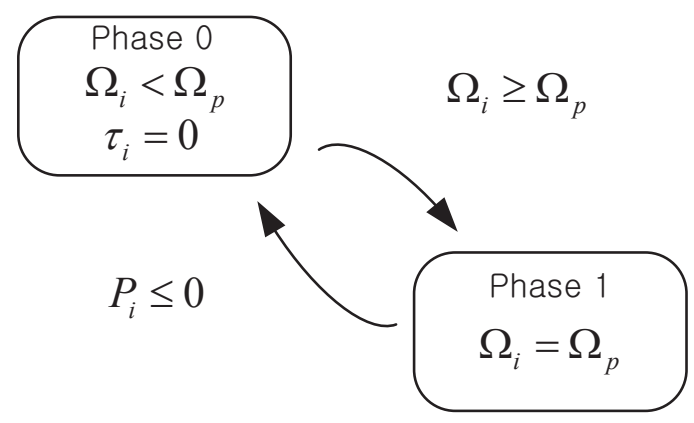

Figure 5. Diagram of the ideal ratchet modeling.

However, it is not easy to implement this modeling in the simulation, even when assumed with extremely short sampling time in the time domain analysis. At the moment of state transition, it must be concurred that $\omega_{i}$ and $P_{i}$ change from some positive to zero, which are the modeling errors.

To overcome this difficulty, we present a ratchet modeling with the addition of the spring element, as shown in Figure 6. Let us define the angle difference between the pulleys $i$ and $p$ as $\Delta Q_{i}(t)=Q_{i}(t)-Q_{p}(t)$ and also define $\Delta Q_{i}\left(t^{*}\right)$ when $t^{*}$ is the moment that the phase changes from 0 to $1\left(\omega_{i}\right.$ outpaces $\left.\omega_{p}\right)$. Then, from this moment $t^{*}$, we assume that the pulleys $i$ and $p$ are connected by virtual torsion spring, and the torque between them is defined as:

$$
\tau_{i}=k_{r}\left\{\Delta Q_{i}(t)-\Delta Q_{i}\left(t^{*}\right)\right\},
$$

where $k_{r}$ is the virtual torsion spring coefficient. 


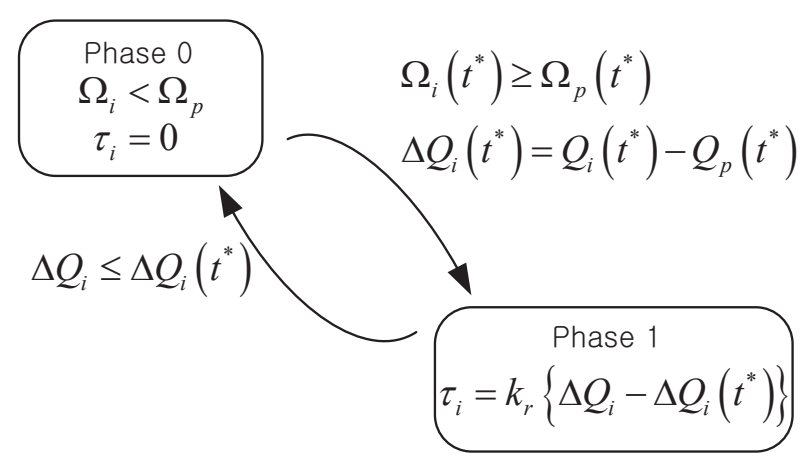

Figure 6. Diagram of the ratchet modeling with the spring element.

As long as $\Delta Q_{i}(t)$ is greater than $\Delta Q_{i}\left(t^{*}\right), \tau_{i}$ is transmitted; otherwise, the phase changes from 0 to 1 , and $\tau_{i}$ becomes zero. This modeling distinguishes the phases via the relation between $\Delta Q_{i}(t)$ and its certain value at the specific moment $\Delta Q_{i}\left(t^{*}\right)$, but does not force the identification between $\omega_{i}$ and $\omega_{p}$, unlike the modeling without the spring element. At the phase transition moment, $\omega_{i}$ must be greater than $\omega_{p}$, but the gap between them is getting smaller due to the spring effect. Hence, there is no discontinuity in the $\omega_{i}$ resulting in no impulse torque that provides the stability in the simulation. It is emphasized that the power (or torque) transmission propensity is dependent on the spring coefficient $k_{r}$.

Let us analyze the effect of $k_{r}$ through the comparison of simple simulations by assigning arbitrary parameters and rope tensions in the PTO module. In Figure 4, the masses of counterweights $m_{1}$ and $m_{2}$ are assumed to be $200 \mathrm{~kg} ; r_{1}$ and $r_{2}$ are $1 \mathrm{~m} ; \kappa=1$; and $J_{g}$ is $0.1 \mathrm{~kg} \cdot \mathrm{m}^{2}$. Then, tensions on the ropes from the buoy are applied as:

$$
\begin{array}{r}
f_{1}=2 \times 10^{3} \sin \left(\frac{2 \pi}{5} t\right)+m_{1} g \\
f_{2}=2 \times 10^{3} \sin \left(\frac{2 \pi}{5} t+1.5\right)+m_{2} g .
\end{array}
$$

By combining (12) and (17) and substituting the assumed parameters and tensions, we can obtain the simulation results as seen in Figures 7 and 8 that $k_{r}$ are set to 10 and $10^{3}$, respectively. When $k_{r}=10$, it can bee seen that $\omega_{p}$ does not track $\omega_{i}$, and power from the generator $P_{g}$ also does not track the trajectory of larger value of $P_{1}$ and $P_{2}$. Otherwise, when $k_{r}=10^{3}$, it results that $\omega_{p}$ can track the trajectory of larger value of $\omega_{1}$ and $\omega_{2}$, and the graph for power provides the equivalent result. The average ratchet efficiency $\eta_{r}$ defined in (16) according to $k_{r}$ is presented in Figure 9, which $\eta_{r}$ increases as $k_{r}$ increases and approaches almost one with $k_{r}$ beyond $10^{3}$. Furthermore, larger inertia results in slightly lower efficiency, since heavy inertia provides the slow response speed like the small $k_{r}$ effect, as seen in Figure 7. The actual inertia, including generator rotor, shaft and gear ratio, is about $\kappa^{2} J_{g}=248 \mathrm{~kg} \cdot \mathrm{m}^{2}$. Hence, $k_{r}$ in the following simulation will be set to $10^{4}$ in order to ignore the power loss in the ratchet gears. 

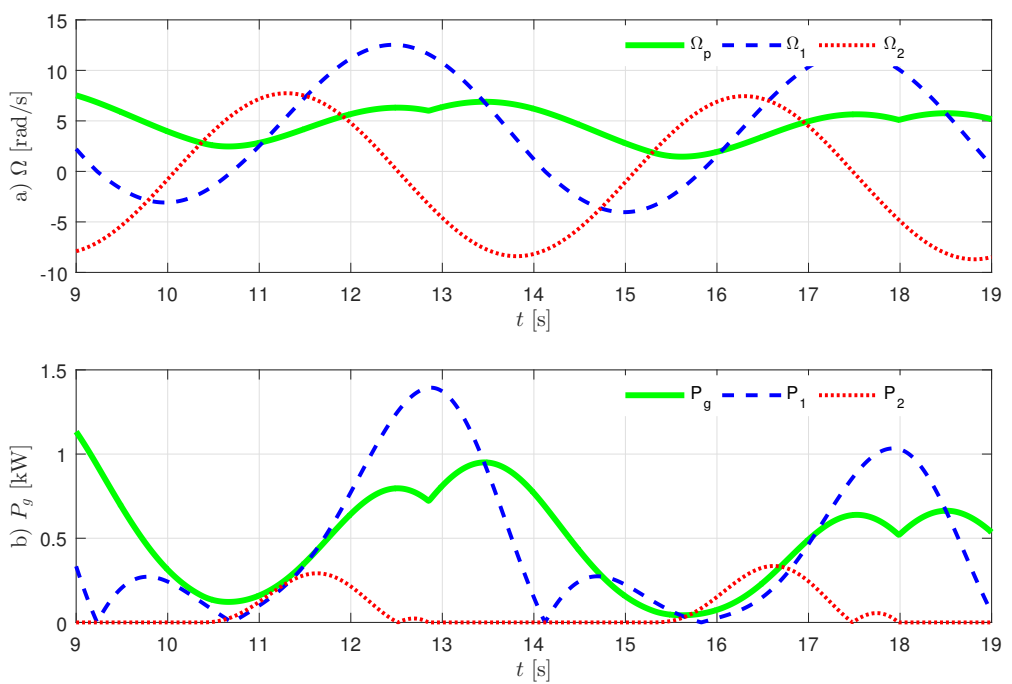

Figure 7. Results of angular speed and power when $k_{r}=10$.
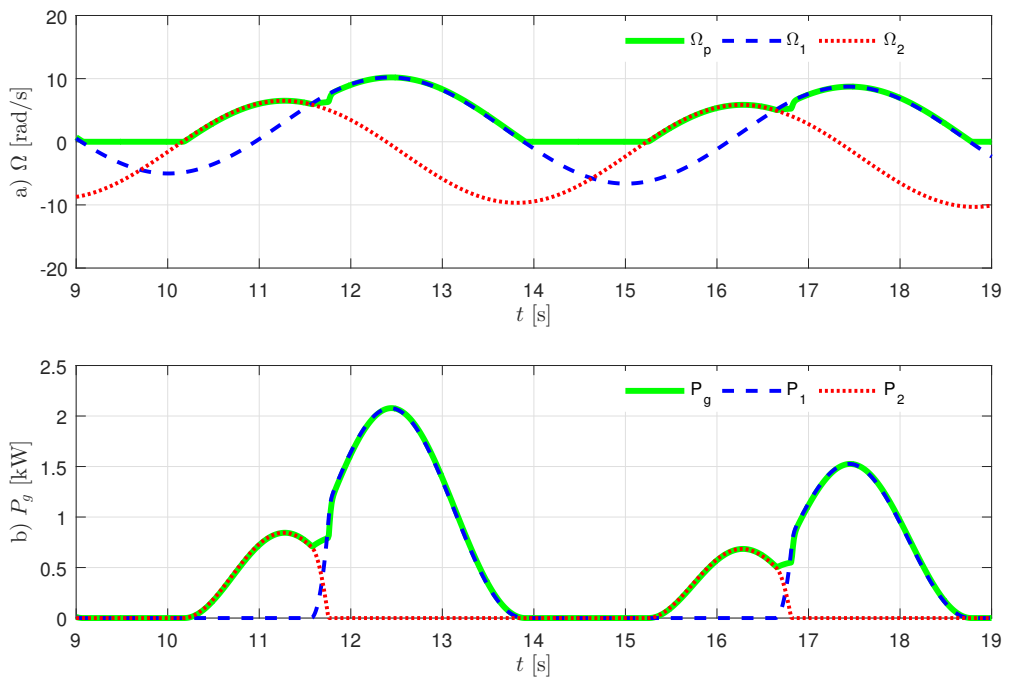

Figure 8. Results of angular speed and power when $k_{r}=10^{3}$.

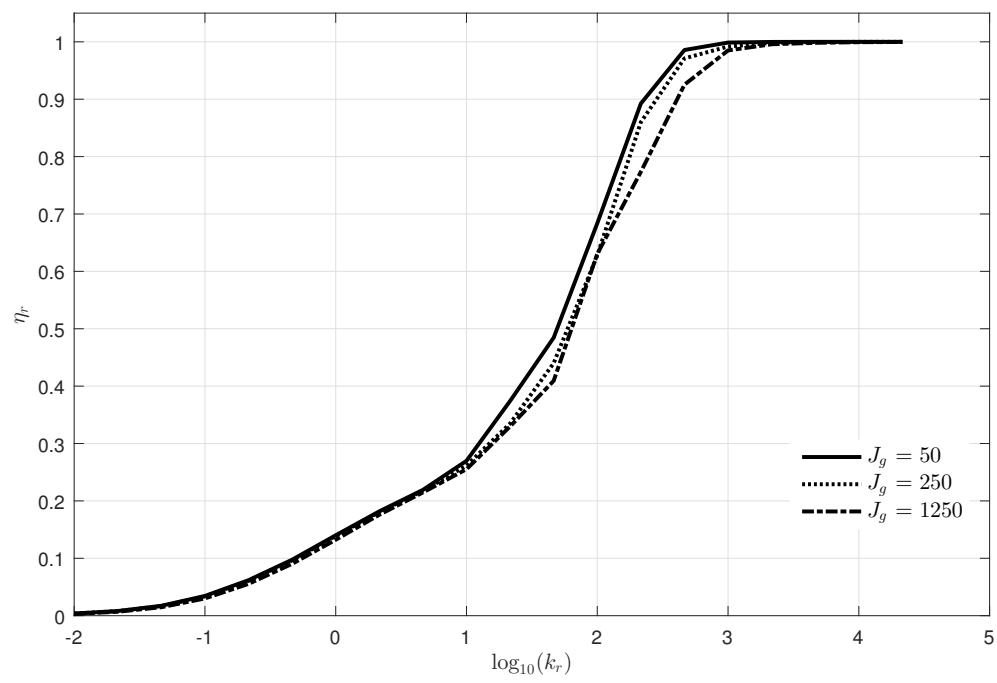

Figure 9. Simulation result of $\eta_{r}$ according to $k_{r}$. 


\subsection{JONSWAP Wave Spectrum Model and Capture Width Ratio}

For irregular wave input, typical wave spectra include JONSWAP, Pierson-Moskowitz, Bretschneider and the Gaussian distribution [13]. We chose the JONSWAP wave model since the area where the INWAVE device has been installed has $\gamma=2.72$ of the JONSWAP spectrum [14]. When the significant wave height is $H_{s}$ and the peak period is $T_{p}\left(=2 \pi / \omega_{p}\right)$, it is expressed in the frequency domain with wave angular frequency $\omega$ as:

$$
S_{\text {JONSWAP }}(\omega)=\frac{5}{16} H_{s}^{2}(1-0.287 \ln \gamma) \frac{\omega_{p}^{4}}{\omega^{5}} \exp \left(-\frac{5}{4}\left(\frac{\omega}{\omega_{p}}\right)^{-4}\right) \gamma^{\exp \left(-0.5\left(\frac{\omega-\omega_{p}}{\sigma \omega p}\right)^{2}\right),}
$$

where:

$$
\sigma=\left\{\begin{array}{l}
0.07 \text { for } \omega \leq \omega_{p} \\
0.09 \text { for } \omega>\omega_{p}
\end{array} .\right.
$$

Then, the wave power per unit crest length is defined as:

$$
J_{\text {JONSWAP }}=\frac{\rho g}{2 \pi} \int_{0}^{\infty} S_{\text {JONSWAP }}(\omega) v_{g}(\omega) d \omega,
$$

where $v_{g}$ is the group velocity given by:

$$
v_{g}=\frac{1}{2}\left[1+\frac{2 k h}{\sinh (2 k h)}\right] v_{p}
$$

and $v_{p}$ is the phase velocity:

$$
v_{p}=\sqrt{\frac{g}{k} \tanh (k h)}
$$

Here, the wave number $k=2 \pi / \lambda$ ( $\lambda$ : the wave length) is obtained from:

$$
\omega^{2}=g k \tanh (k h) .
$$

We also define the normalized wave power per unit crest length and unit wave amplitude as:

$$
J_{\text {JONSWAP }}^{*}=\frac{J_{\text {JONSWAP }}}{\left(H_{S} / 2\right)^{2}}
$$

This is only a function of the wave period and can be seen in Figure 10 with $h=5 \mathrm{~m}$, which is the water depth of the installation area.

In order to quantify the power performance with the consideration of the device's dimension, the capture width ratio (CWR) $\eta_{1}$ is widely used and is defined as follows [15]:

$$
\eta_{1}=\frac{\bar{P}_{g}^{*}}{J_{\text {JONSWAP }}^{*} B^{\prime}},
$$

where $B=\sqrt{4 A_{w} / \pi}$ is the characteristic dimension and $A_{w}$ is the maximum horizontal cross-sectional area of the device. In this paper, for a cylindrical buoy, $A_{w}$ is simply equal to $\pi R^{2}$. Hence, $B=2 R$ where $R$ is the radius of the buoy. 


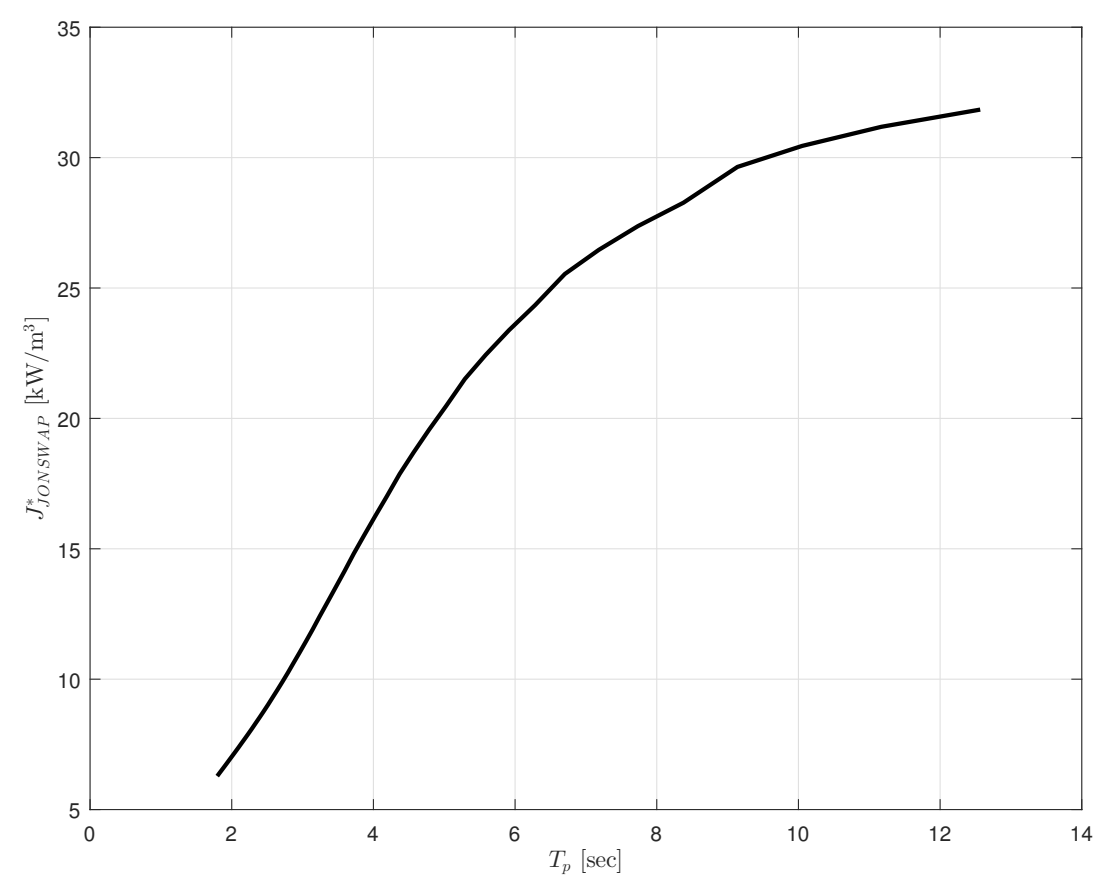

Figure 10. JONSWAP with varying $T_{p}$ when $h=5 \mathrm{~m}$.

\section{Simulation in the Time Domain}

In this section, simulation of the buoy and PTO combined dynamics is carried out in the time domain under the irregular wave condition with the JONSWAP wave model [16]. For time domain analysis, the Runge-Kutta method was applied for solving the dynamics [17].

We obtained the hydrodynamic parameters of the buoy from AQWA (version 14, ANSYS, Pittsburgh, PE, USA) [18]. In order to calculate kernel function of excitation force $f_{e}(t)$ and radiation damping $f_{r}(t)$, the frequency domain responses for excitation forces $\hat{f}_{e}(\omega)$, radiation $\hat{R}(\omega)$ and added mass $\mu(\omega)$ need to be calculated. In the AQWA simulation, the buoy has a $2 R=5 \mathrm{~m}$ for diameter, with $0.5 \mathrm{~m}$ of draft, which reflects the actual size of the prototype buoy. It is floating in the ocean cube that has a 500 by $500 \mathrm{~m}^{2}$ surface and $h=5 \mathrm{~m}$ of water depth, which is the actual depth of the installation area, and only surge, heave and pitch motions of the body are allowed. The frequency domain response should have a truncation frequency of $5 \mathrm{rad} / \mathrm{s}$ with a frequency spacing of $0.05 \mathrm{rad} / \mathrm{s}$ [19].

The lines in Figure 11 display the kernel functions of excitation force $f_{e}(t)$ and radiation damping force $f_{r}(t)$ from the calculations of (7) and (9), respectively.

A JONSWAP wave model is adapted in order to generate irregular waves in the simulation. For time series calculations, the spectral distribution (18) is discretized as the sum of a large number $M$ of regular waves, written as:

$$
\psi(t)=\sum_{m=1}^{M} \psi_{m} \cos \left(\omega_{m} t+\theta_{m}\right)
$$

Here, $\omega_{m}=\omega_{l}+(m-1) \Delta \omega$, where $\omega_{l}$ is the lowest frequency, $\Delta \omega$ is a small frequency interval, $m=1,2, \ldots, M$ and the spectrum does not contain a significant amount of energy outside the frequency range $\omega_{l} \leq \omega \leq \omega_{l}+(M-1) \Delta \omega . \psi_{m}=\sqrt{2 S_{\text {JONSWAP }}\left(\omega_{m}\right) \Delta \omega}$ and $\theta_{m}$ are the amplitude of the wave component of order $m$ and the initial phase randomly chosen in the interval $(0,2 \pi)$, respectively. 

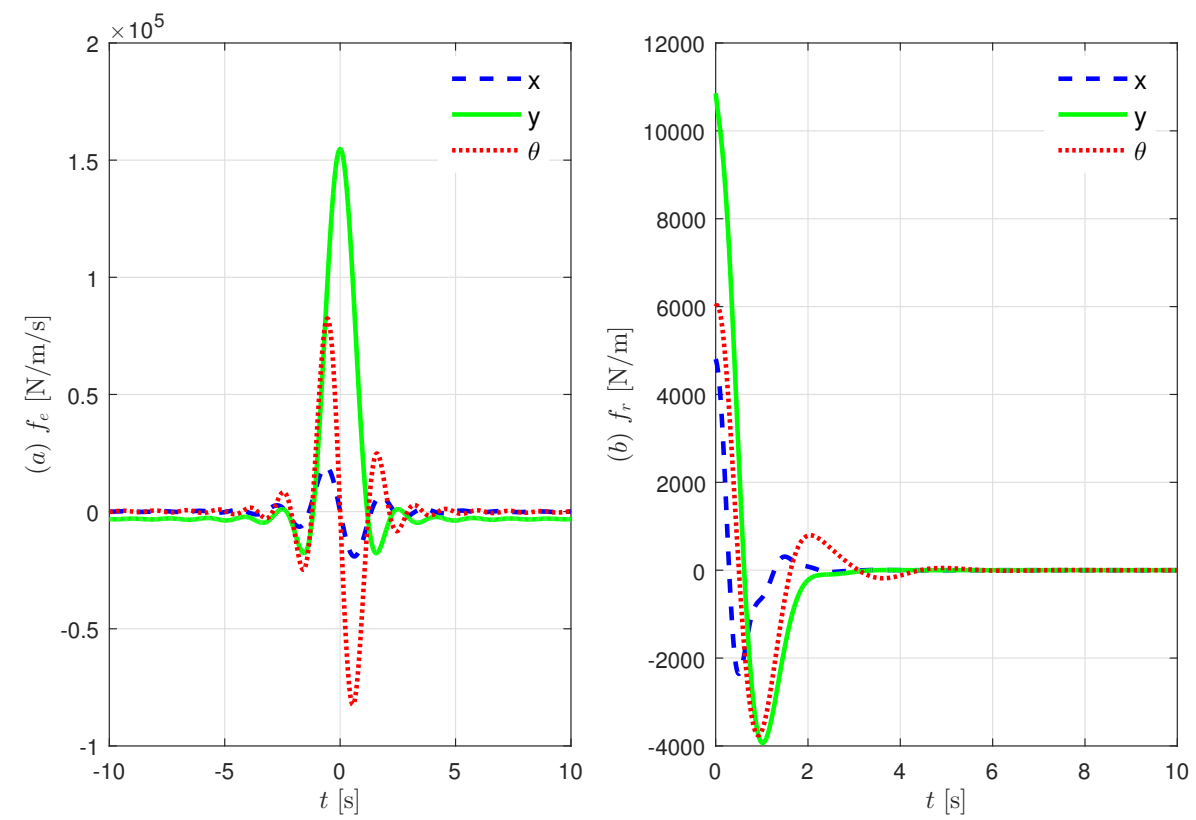

Figure 11. Graphs of kernel functions: (a) excitation force; (b) radiation damping force.

With the excitation force vector formed by substituting (22) into (6), the combined dynamics with (11)-(13) and (17) was performed according to the Runge-Kutta method with $0.001 \mathrm{~s}$ sampling time [17]. A summary of the parameters and values used in the simulations is given in Table 1. Figure 12 displays the result of wave elevation, the buoy's posture, the angular speed of pulleys, the tension of ropes and the power output from the generator in the time domain when $T_{p}=4.5 \mathrm{~s}, H_{s}=1$ $\mathrm{m}$ and $b_{g}=5 \mathrm{Nm} \mathrm{s} / \mathrm{rad}$.

Table 1. Parameters and values in the simulations.

\begin{tabular}{ccc}
\hline Parameter & Value & Unit \\
\hline$\rho_{w}$ & 1025 & $\mathrm{~kg} / \mathrm{m}^{3}$ \\
$R$ & 2.5 & $\mathrm{~m}$ \\
$r$ & 1.5 & $\mathrm{~m}$ \\
$h$ & 5 & $\mathrm{~m}$ \\
$D$ & 3 & $\mathrm{~m}$ \\
$m_{1}, m_{2}$ & 250 & $\mathrm{~kg}$ \\
$m_{b}$ & 2500 & $\mathrm{~kg}$ \\
$J_{b}$ & 3906 & $\mathrm{~kg} \cdot \mathrm{m}^{2}$ \\
$r_{1}, r_{2}$ & 0.25 & $\mathrm{~m}$ \\
$\kappa$ & 35 & - \\
$k_{r}$ & $10^{4}$ & $\mathrm{~N} / \mathrm{rad}$ \\
\hline
\end{tabular}

In Figure 12, irregular waves yield complex motions of surge, heave and pitch. Furthermore, angular velocities of pulleys connected to the ropes and the generator also have very complex trajectories. The pulley speed $\omega_{p}$ somewhat tracks the positive max value of $\omega_{1}$ and $\omega_{2}$ with small error. Finally, as $\omega_{p}$ increases, the power from generator $P_{g}$ also increases. Tensions $T_{1}$ and $T_{2}$ are only 0.25 tons of counterweight when power is not transmitted, and $P_{g}$ is generated as tension increases. During the 30-min duration of the simulation, the average power output is $10.56 \mathrm{~kW}$.

The power output varies sensitively in accordance with $b_{g}$. Figure 13 displays the graphs of $\bar{P}_{g}^{*}$ with varying $b_{g}$ under irregular waves with $T_{p}=4.5 \mathrm{~s}$. Let us define $\bar{P}_{g}^{*}$ and $b_{g}$ as $\bar{P}_{g, o p t}^{*}$ and $b_{g, o p t}$, respectively, at the peak of the power output. In Figure 13, it can be seen that $\bar{P}_{g}^{*}$ rapidly increases when $b_{g}<b_{g, o p t}, \bar{P}_{g, o p t}^{*}=33.8 \mathrm{~kW}$ at $b_{g, o p t}=3.8$ and gradually decreases when $b_{g}>b_{g, o p t}$. 

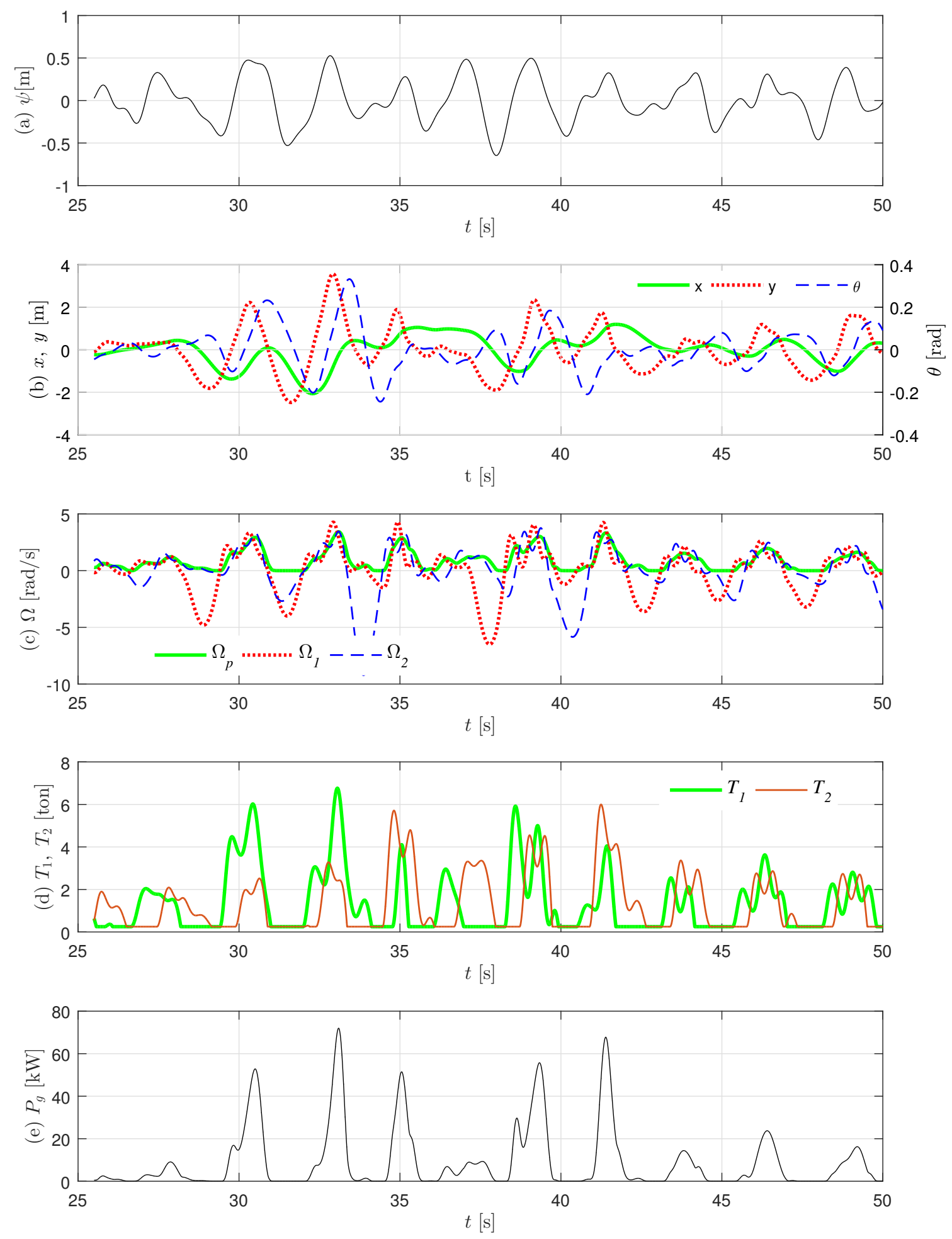

Figure 12. Simulation result of the INWAVE device under the JONWSAPwave model with $H_{s}=1 \mathrm{~m}$ and $T_{p}=4.5 \mathrm{~s}$ when $b_{g}=5 \mathrm{Nm} \mathrm{s} / \mathrm{rad}$ : (a) wave elevation; (b) buoy's posture; (c) pulley's angular speed; (d) rope tension; (e) power from the generator. 


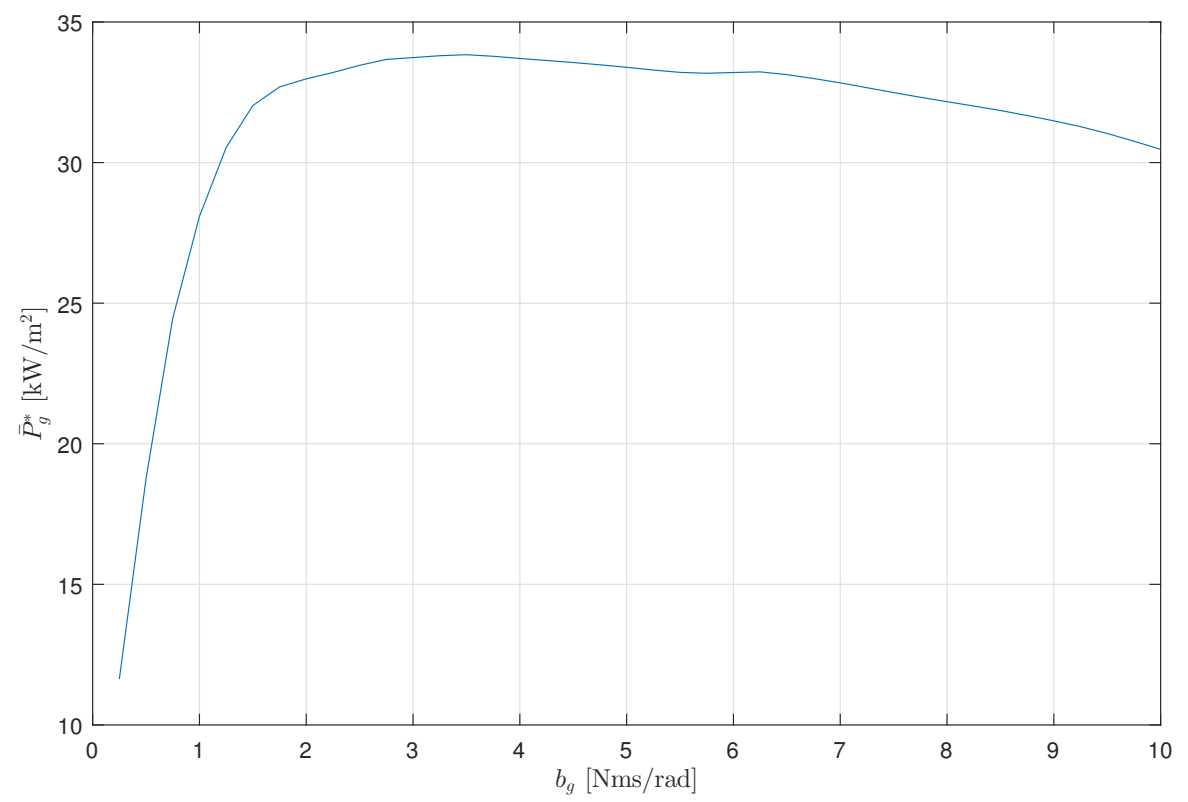

Figure 13. Graph of $\bar{P}_{g}^{*}$ with varying $b_{g}$ when $T_{p}=4.5 \mathrm{~s}$.

According to the LWT, the normalized power output is mainly a function of $T_{p}$ and $b_{g}$. We calculate $\bar{P}_{g, o p t}^{*}$ for varying $T_{p}$, and Figure 14 shows the results of the $\bar{P}_{g, o p t}^{*}$ and $b_{g, o p t}$ for varying $T_{p}$ between 2.5 and $12 \mathrm{~s}$. It can be seen that $\bar{P}_{g, o p t}^{*}$ has $70 \mathrm{~kW} / \mathrm{m}^{2}$ of maximum output at $T_{p}=2.7 \mathrm{~s}$, and the maximum value of $C W R \eta_{1}$ reaches almost 1.645 at $T_{p}=2.31 \mathrm{~s}$. The optimal damping coefficient $b_{g, o p t}$ increases with $2<T_{p}<3.5$ and $4.9<T_{p}<6.2 \mathrm{~s}$ and the levels between them, but drastically decreases when $T_{p}>6.5 \mathrm{~s}$. Since our targeted installation area has the $T_{p}$ between 2.5 and $4.3 \mathrm{~s}$, the simulation that displays the maximum power between 2 and $4 \mathrm{~s}$ of $T_{p}$, we can see that the design of the INWAVE somewhat meets the design direction.
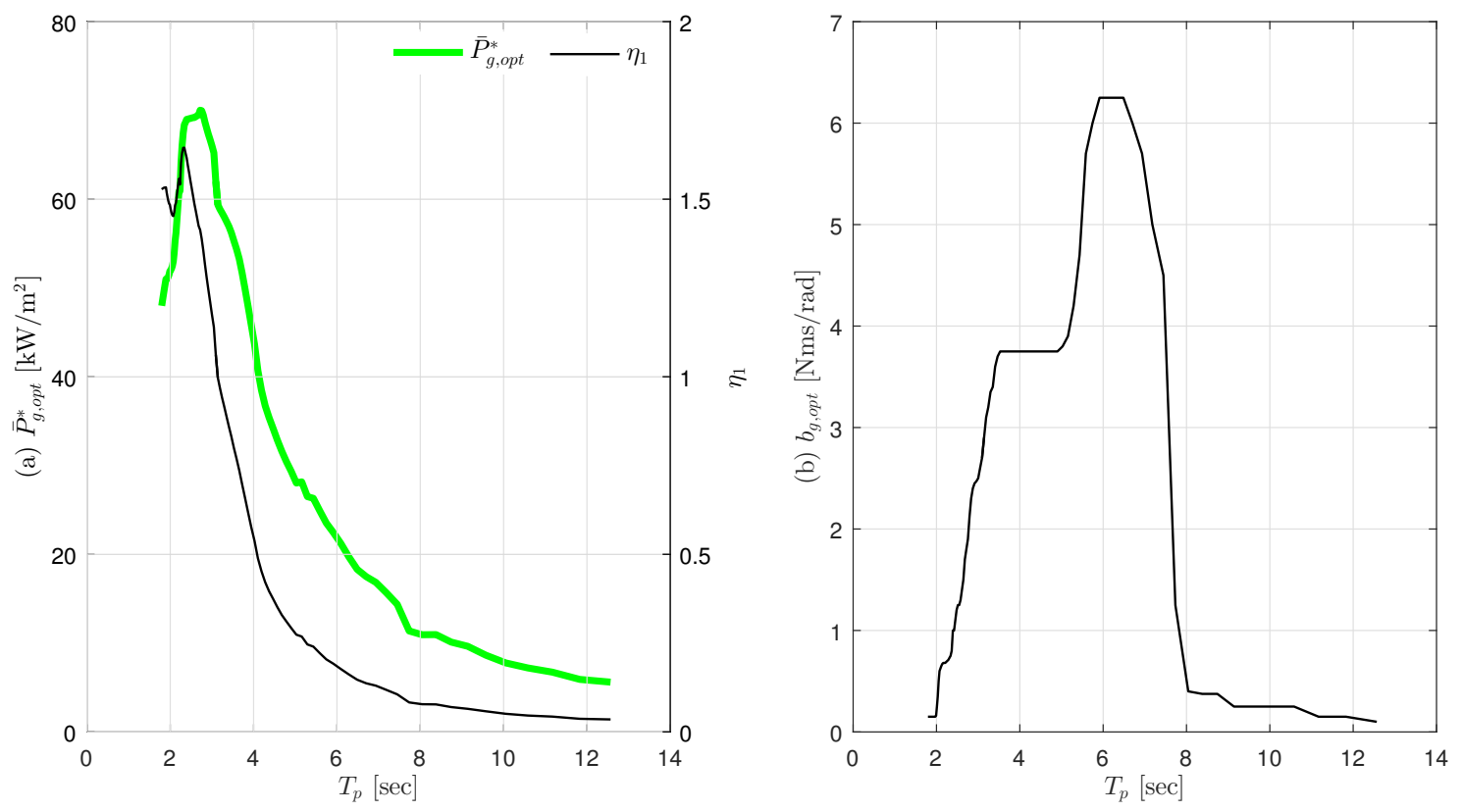

Figure 14. Graphs of $\bar{P}_{g, o p t}^{*}$ and $b_{g, o p t}^{*}$ with varying $T_{p}$. 


\section{Characteristics of the INWAVE Device}

Commonly, a point absorber is installed in an area deeper than $20 \mathrm{~m}$ of water. CETO [20] and the Lysekil project [21] pick the center of the buoy as the single point mooring and connect it to PTO because these locations have high enough energy density to concentrate mainly on the heave motions. As water depths become shallower, the wave moves more in the direction of the surge than heave. As such, the INWAVE device focuses on the surge and pitch motion and is installed nearer to the coast. Waves in shallow water are affected by sea-bed topography, resulting in irregular motions. To overcome this, the INWAVE device is connected via three ropes that are on the bottom of the buoy. It is designed to respond to all motions within six degrees of freedom. The three ropes that are connected to the buoy are also connected to the counterweight on land via underwater pulleys. The ropes also spin the rope drum. Each rope drum transmits the torque to the electrical generator through the ratchet mechanism. While all three ropes move back and forth irregularly according to the movement of the buoy, they do not move in phase with each other. Accordingly, the time point in which each rope drum sends power to the generator is different. This reduces the effect of the inhomogeneity of electricity, which is one of disadvantages of wave power energy.

Ropes are preferentially considered when designing the device. Due to the tension that is always in action between the buoy and the counterweight, ropes must have toughness to endure stress, the ability to endure salinity and sunlight, as well as the friction created as it winds up on the underwater pulley and the rope drums. While this paper's analysis was done with the assumption that power is supplied to the generator only when the buoy pulls on the rope and the counterweight rises, in reality, torque can be supplied to the generator when the counterweight is descending, as well. For this to occur, the ratchet mechanism needs to be designed in such a way that it allows for the generator to spin in one direction, regardless of the direction of the counterweight's movement. Currently, both ratchet mechanisms are undergoing field tests. In terms of smoothing of the created electricity, the best method would be to send all of the power created by two-way directional spin. However, to do so, we must carefully consider the relationship between the mass of the counterweight and the maximum wave height. The greatest function of the counterweight is providing the buoy with restorability. While heave and pitch motion gets restorability from buoyancy, surge motion gets its restorability from the tensions supplied from the rope by the counterweight. If the counterweight sends force to the generator on its way down, the tension supplied to the buoy will inevitably be smaller. If the tension supplied becomes zero, the rope will become loose. The moment the counterweight rises, it will put sudden shock on the rope, causing the rope to potentially break, or breakaway from the rope drum. This would not be a problem if the mass of the counterweight is sufficient. However, this would require the rope to be tougher, so that it can endure higher tension. Thus, we did not simulate this kind of situation because it would require more complex hardware. However, in future works, we plan to compare and analyze the data given by the prototypes.

\section{Conclusions}

This paper introduced the INWAVE device developed by INGINE Inc. In order to evaluate its theoretical performance, dynamic analysis based on the Newtonian mechanics was derived in the two dimensions, and the time domain simulation was carried out under the LWT. The parameters used in the simulation were obtained by using the ANSYS AQWA simulator and inverse Fourier transform. In order to overcome the dynamic discontinuity of the ratchet gear mechanism in the time domain simulation, we suggest the modeling of the ratchet gear by introducing the virtual torsion spring element and prove its efficiency under various generator inertia and torsion spring coefficients. In the simulation, the JONSWAP wave model with $\gamma=2.72$ was adapted to generate irregular waves, to ensure that the model is reflecting the actual installation area. The simulation results provide the theoretical capture width ratio with the optimal operation condition. There are two limitations in the paper; the LWT was applied at a low water depth condition, and the effect of viscous damping was ignored. If the low depth and viscous damping were considered, the CWR would have been lower. 
In addition, as mentioned in the previous section, the physical limitation of the rope, the non-linearity of the generator (including the inverter process) and friction loss with the threshold of the mechanical components should be addressed.

Despite the inaccuracies of the buoy's hydrodynamic modeling, this modeling has become a reference for practical design decisions. This is because the modeling uncertainty can be offset by multiplying the safety margin factor. For example, we have found the design criteria for rope tension that determine the thickness of the rope, the diameter of the rope drum and the diameter of the shaft in the PTO module. In addition, a relationship between the generator's torque limit and the inertia of the gear module on the power output has been researched, which is reflected in the design of the next prototype. We will present the three-dimensional modeling analysis and discuss the optimization of mechanical elements including the hardware limitations in the future.

Acknowledgments: The presented work was supported by INGINE Inc.

Author Contributions: Y.J.S made a device; S.K.S. conceived and designed the simulations; S.K.S. performed the simulations; S.K.S. and J.B.P. analyzed the data; S.K.S wrote the paper.

Conflicts of Interest: The authors declare no conflict of interest.

\section{References}

1. Johansson, T.B. Renewable Energy: Sources for Fuels and Electricity; Island Press: Washington, DC, USA, 1993.

2. Lindroth, S.; Leijon, M. Offshore wave power measurements-A review. Renew. Sustain. Energy Rev. 2011, 15, 4274-4285.

3. Mørk, G.; Barstow, S.; Kabuth, A.; Pontes, M.T. Assessing the global wave energy potential. In Proceedings of the 29th International Conference on Ocean, Offshore and Arctic Engineering, Shanghai, China, 6-11 June 2010.

4. Falnes, J. Ocean Waves and Oscillating Systems: Linear Interactions Including Wave-Energy Extraction; Cambridge University Press: Cambridge, UK, 2002.

5. Clément, A.; McCullen, P.; Falcão, A.; Fiorentino, A.; Gardner, F.; Hammarlund, K.; Lemonis, G.; Lewis, T.; Nielsen, K.; Petroncini, S.; et al. Wave energy in Europe: Current status and perspectives. Renew. Sustain. Energy Rev. 2002, 6, 405-431.

6. Nielsen, S.N.; Jørgensen, S.E. Sustainability analysis of a society based on exergy studies-A case study of the island of Samsø (Denmark). J. Clean. Prod. 2015, 96, 12-29.

7. Yong Jun, S. Available online: https:/ /www.youtube.com/watch?v=-vt2PJdBhkU (accessed on 14 October 2016).

8. Sjolte, J.; Sandvik, C.M.; Tedeschi, E.; Molinas, M. Exploring the potential for increased production from the wave energy converter lifesaver by reactive control. Energies 2013, 6, 3706-3733.

9. Lattanzio, S.; Scruggs, J. Maximum power generation of a wave energy converter in a stochastic environment. In Proceedings of the 2011 IEEE International Conference on Control Applications (CCA), Denver, CO, USA, 28-30 September 2011; IEEE: New York, NY, USA, 2011; pp. 1125-1130.

10. Cummins, W.E. The Impulse Response Function and Ship Motions; Technical Report, DTIC Document; David Taylor Model Basin: Washington, DC, USA, 1962.

11. Dean, R.G.; Dalrymple, R.A. Water Wave Mechanics for Engineers and Scientists; World Scientific Publishing Company: Singapore, 1991.

12. Budar, K.; Falnes, J. A resonant point absorber of ocean-wave power. Nature 1975, 256, 478.

13. Holmes, B. Tank Testing of Wave Energy Conversion Systems: Marine Renewable Energy Guides; European Marine Energy Centre: Orkney, UK, 2009.

14. Kang, D.H.; Lee, B.G. Evaluation of Wave Characteristics and JONSWAP Spectrum Model in the Northeastern Jeju Island on Fall and Winter. J. Korean Soc. Mar. Environ. Energy 2014, 17, 63-69.

15. Babarit, A. A database of capture width ratio of wave energy converters. Renew. Energy 2015, 80, 610-628.

16. Hasselmann, K.; Barnett, T.; Bouws, E.; Carlson, H.; Cartwright, D.; Enke, K.; Ewing, J.; Gienapp, H.; Hasselmann, D.; Kruseman, P.; et al. Measurements of Wind-Wave Growth and Swell Decay during the Joint North Sea Wave Project (JONSWAP); Technical Report; Deutsches Hydrographisches Institut: Hamburg, Germany, 1973. 
17. Butcher, J.C. The Numerical Analysis of Ordinary Differential Equations: Runge-Kutta and General Linear Methods; Wiley-Interscience: Hoboken, NJ, USA, 1987.

18. Ansys, A. User Manual v.13.0, 2010; Century Dynamics Ltd.: Horsham, UK, 2010.

19. Bosma, B.; Zhang, Z.; Brekken, T.K.A.; Özkan-Haller, H.T.; McNatt, C.; Yim, S.C. Wave energy converter modeling in the frequency domain: A design guide. In Proceedings of the 2012 IEEE Energy Conversion Congress and Exposition (ECCE), Raleigh, NC, USA, 15-20 September 2012; IEEE: New York, NY, USA, 2012; pp. 2099-2106.

20. Mann, L.; Burns, A.; Ottaviano, M. CETO, a carbon free wave power energy provider of the future. In Proceedings of the 7th European Wave and Tidal Energy Conference (EWTEC), Porto, Portugal, 11-13 September 2007.

21. Leijon, M.; Boström, C.; Danielsson, O.; Gustafsson, S.; Haikonen, K.; Langhamer, O.; Strömstedt, E.; Stålberg, M.; Sundberg, J.; Svensson, O.; et al. Wave energy from the North Sea: Experiences from the Lysekil research site. Surv. Geophys. 2008, 29, 221-240.

(C) 2017 by the authors; licensee MDPI, Basel, Switzerland. This article is an open access article distributed under the terms and conditions of the Creative Commons Attribution (CC-BY) license (http://creativecommons.org/licenses/by/4.0/). 